\title{
ON $k$-CIRCULANT MATRICES INVOLVING THE JACOBSTHAL NUMBERS
}

\author{
BILJANA RADIČIĆ
}

\begin{abstract}
Let $k$ be a nonzero complex number. We consider a $k$-circulant matrix whose first row is $\left(J_{1}, J_{2}, \ldots, J_{n}\right)$, where $J_{n}$ is the $n^{\text {th }}$ Jacobsthal number, and obtain the formulae for the eigenvalues of such matrix improving the formula which can be obtained from the result of Y. Yazlik and N. Taskara [J. Inequal. Appl. 2013, 2013:394, Theorem 7]. The obtained formulae for the eigenvalues of a $k$-circulant matrix involving the Jacobsthal numbers show that the result of Z. Jiang, J. Li, and N. Shen [WSEAS Trans. Math. 12 (2013), no. 3, 341-351, Theorem 10] is not always applicable. The Euclidean norm of such matrix is determined. We also consider a $k$-circulant matrix whose first row is $\left(J_{1}^{-1}, J_{2}^{-1}, \ldots, J_{n}^{-1}\right)$ and obtain the upper and lower bounds for its spectral norm.
\end{abstract}

\section{INTRODUCTION}

Throughout this paper $k$ is a nonzero complex number.

Definition 1.1. A $k$-circulant matrix with the first row $\left(c_{0}, c_{1}, c_{2}, \ldots, c_{n-1}\right)$ is a square matrix having the following form:

$$
\left[\begin{array}{cccccc}
c_{0} & c_{1} & c_{2} & \ldots & c_{n-2} & c_{n-1} \\
k c_{n-1} & c_{0} & c_{1} & \ldots & c_{n-3} & c_{n-2} \\
k c_{n-2} & k c_{n-1} & c_{0} & \ldots & c_{n-4} & c_{n-3} \\
\vdots & \vdots & \vdots & \ddots & \vdots & \vdots \\
k c_{2} & k c_{3} & k c_{4} & \ldots & c_{0} & c_{1} \\
k c_{1} & k c_{2} & k c_{3} & \ldots & k c_{n-1} & c_{0}
\end{array}\right]
$$

Necessary and sufficient conditions for a complex square matrix to be a $k$-circulant matrix were presented in the paper [4] (see [4, Lemmas 2 and 3]). Namely, $k$-circulant matrices present a special case of Toeplitz matrices-matrices having constant main diagonals. For more information about Toeplitz matrices, we recommend [5, 6, 17, 13, 17, 25].

2010 Mathematics Subject Classification. Primary 15B05; Secondary 11B37, 15A09, 15A18, 15A60.

Key words and phrases. $k$-circulant matrix; Jacobsthal numbers; Eigenvalues; Norms of a matrix; Hadamard inverse of a matrix. 
We shall write $\operatorname{circ}_{n}\left\{k_{k}\left(c_{0}, c_{1}, c_{2}, \ldots, c_{n-1}\right)\right\}$ for a $k$-circulant matrix (of order $n$ ) whose first row is $\left(c_{0}, c_{1}, c_{2}, \ldots, c_{n-1}\right)$. If the order of a matrix is known, then the designation for the order of a matrix can be omitted. If $k=1$, then $k$-circulant matrices are called circulant matrices. If $k=-1$, then $k$-circulant matrices are called skew circulant matrices.

It should be pointed out that, in recent years, $k$-circulant matrices present one of the most important research fields of applied mathematics and computational mathematics. We recommend the following papers devoted to $k$-circulant matrices: $[3,4,9,14,21,22,23,24,26]$. Let us mention that $k$-circulant matrices have a wide range of applications in many areas such as signal and image processing, probability, statistics, numerical analysis, economy, coding theory, and engineering modeling. In the papers [1, 8, 19, 20, 27] the authors describe some of their applications.

For any complex matrix $C$ of order $n$, the symbols $\lambda_{j}(C)(j=\overline{0, n-1}),|C|$, $C^{\circ-1},\|C\|_{E}$, and $\|C\|_{2}$ denote the eigenvalues, the determinant, the Hadamard inverse (provided that $c_{i, j} \neq 0$ for all $i, j=\overline{1, n}$ ), the Euclidean norm, and the spectral norm of $C$, respectively.

Definition 1.2. Let $C=\left[c_{i, j}\right]$ be a complex matrix of order $n$.

(1) $C^{\circ-1}=\left[c_{i, j}^{-1}\right]$, if $c_{i, j} \neq 0$ for all $i, j=\overline{1, n}$;

(2) $\|C\|_{E}=\sqrt{\sum_{i, j=1}^{n}\left|c_{i, j}\right|^{2}}$;

(3) $\|C\|_{2}=\sqrt{\max _{1 \leq i \leq n} \lambda_{i}\left(C^{*} C\right)}$, where $C^{*}$ is the conjugate transpose of $C$.

Let us also mention that the following inequalities hold for any complex matrix $C$ of order $n$ (see [28, Theorem 1 and Table 1]):

$$
\frac{\|C\|_{E}}{\sqrt{n}} \leq\|C\|_{2} \leq\|C\|_{E} .
$$

In this paper, we consider the matrix

$$
J=\operatorname{circ}\left\{k\left(J_{1}, J_{2}, \ldots, J_{n}\right)\right\},
$$

where $J_{n}$ is the $n^{\text {th }}$ Jacobsthal number. The motivation for this paper was found in the papers [2], 14 and 26]. Namely, we shall improve the result in relation to the eigenvalues of $(1.2)$ which can be obtained from the formula for the eigenvalues of a $k$-circulant matrix with the generalized $r$-Horadam numbers $\left\{H_{r, n}\right\}$ (the numbers defined as follows:

$$
H_{r, n+2}=f(r) H_{r, n+1}+g(r) H_{r, n}, \quad n \geq 0,
$$

where $r \in \mathbb{R}^{+}, H_{r, 0}=a, H_{r, 1}=b, a, b \in \mathbb{R}$, and $\left.f^{2}(r)+4 g(r)>0\right)$, presented in [26], because the authors did not consider the case when the denominator is equal to zero. 
Theorem $1.3\left(\left[26\right.\right.$, Theorem 7]). Let $H=\operatorname{circ}\left\{k_{k}\left(H_{r, 0}, H_{r, 1}, \ldots, H_{r, n-1}\right)\right\}$. The eigenvalues of $H$ are:

$$
\lambda_{j}(H)=\frac{k H_{r, n}+\left(g(r) k H_{r, n-1}-b+a f(r)\right) \psi \omega^{-j}-H_{r, 0}}{g(r)\left(\psi \omega^{-j}\right)^{2}+f(r) \psi \omega^{-j}-1}, \quad j=\overline{0, n-1},
$$

where $\psi$ and $\omega$ are any $n^{\text {th }}$ root of $k$ and any primitive $n^{\text {th }}$ root of unity, respectively.

Our result in relation to the eigenvalues of 1.2 will show that the following theorem is not always applicable.

Theorem 1.4 ([14, Theorem 10]; see also [26, Theorem 8]). Let $J$ be the matrix as in 1.2 . The determinant of $J$ is

$$
|J|=\frac{\left(1-k J_{n+1}\right)^{n}-k\left(2 k J_{n}\right)^{n}}{1-j_{n} k+(-2)^{n} k^{2}},
$$

where $j_{n}$ is the $n^{\text {th }}$ Jacobsthal-Lucas number.

In this paper, we also obtain the Euclidean norm of $(1.2)$ and bounds for the spectral norm of a $k$-circulant matrix whose first row is $\left(J_{1}^{-1}, J_{2}^{-1}, \ldots, J_{n}^{-1}\right)$. Bearing in mind that, for $k=1$, the obtained results are related with a circulant matrix whose first row is $\left(J_{1}, J_{2}, \ldots, J_{n}\right)$, the results of this paper complement the results of the paper [2] where the authors obtained the determinant (without using the formulae for the eigenvalues) and the inverse of such matrix.

Before we present our main results, let us recall that the Jacobsthal numbers $\left\{J_{n}\right\}$ satisfy the following recurrence relation:

$$
J_{n}=J_{n-1}+2 J_{n-2}, \quad n \geq 2,
$$

with initial conditions $J_{0}=0$ and $J_{1}=1$. These numbers were introduced for the first time by the famous German mathematician Ernst Jacobsthal (1882-1965).

The first few terms of the Jacobsthal sequence are given by the following table:

\begin{tabular}{|c|c|c|c|c|c|c|c|c|}
\hline$n$ & 0 & 1 & 2 & 3 & 4 & 5 & 6 & $\ldots$ \\
\hline$J_{n}$ & 0 & 1 & 1 & 3 & 5 & 11 & 21 & $\ldots$ \\
\hline
\end{tabular}

Let $\alpha$ and $\beta$ be the roots of the equation $x^{2}-x-2=0$, i.e., $\alpha=2$ and $\beta=-1$. Therefore,

$$
\alpha \beta=-2, \quad \alpha+\beta=1, \quad \text { and } \quad \alpha-\beta=3 .
$$

From the equation $x^{2}-x-2=0$, it is easy to see that the following equality holds:

$$
x^{n}=J_{n} x+2 J_{n-1} .
$$

Since both $\alpha$ and $\beta$ are roots of the equation $x^{2}-x-2=0$, they must both satisfy $x^{n}=J_{n} x+2 J_{n-1}$. Therefore,

$$
\alpha^{n}=J_{n} \alpha+2 J_{n-1} \quad \text { and } \quad \beta^{n}=J_{n} \beta+2 J_{n-1} .
$$


Subtracting the second equation from the first equation in 1.5 yields Binet's formula for the Jacobsthal numbers:

$$
J_{n}=\frac{\alpha^{n}-\beta^{n}}{\alpha-\beta}=\frac{1}{3}\left(2^{n}-(-1)^{n}\right) .
$$

Let us also recall that the Jacobsthal-Lucas numbers $\left\{j_{n}\right\}$ satisfy the same recurrence relation (as the Jacobsthal numbers) but with initial conditions $j_{0}=2$ and $j_{1}=1$, and

$$
j_{n}=\alpha^{n}+\beta^{n}=2^{n}+(-1)^{n}
$$

is Binet's formula for the Jacobsthal-Lucas numbers.

The following identity holds for the Jacobsthal numbers:

$$
\sum_{i=1}^{n} J_{i}^{2}=\frac{1}{9}\left(2^{n+2} J_{n}+n\right) .
$$

More information about the Jacobsthal numbers can be found in [10] and [16].

Our results will be presented in the following section.

\section{MAin RESUltS}

Before starting to present our results, let us recall that any $n^{\text {th }}$ root of $k$ and any primitive $n^{\text {th }}$ root of unity are denoted by $\psi$ and $\omega$, respectively. We shall use the following lemma presented by R. E. Cline, R. J. Plemmons, and G. Worm in the paper [4].

Lemma 2.1 ([4, Lemma 4]). The eigenvalues of $C=\operatorname{circ}\left\{{ }_{k}\left(c_{0}, c_{1}, c_{2}, \ldots, c_{n-1}\right)\right\}$ are:

$$
\lambda_{j}(C)=\sum_{i=0}^{n-1} c_{i}\left(\psi \omega^{-j}\right)^{i}, \quad j=\overline{0, n-1} .
$$

Moreover, in this case

$$
c_{i}=\frac{1}{n} \sum_{j=0}^{n-1} \lambda_{j}(C)\left(\psi \omega^{-j}\right)^{-i}, \quad i=\overline{0, n-1} .
$$

First, we obtain the eigenvalues of the matrix (1.2).

Theorem 2.2. Let $J$ be the matrix as in 1.2 . The eigenvalues of $J$ are given by the following formulae:

(1) If $\psi \omega^{-j}=\frac{1}{2}$, then

$$
\lambda_{j}(J)=\frac{2}{9}\left(3 n-\left(-\frac{1}{2}\right)^{n}+1\right) .
$$

(2) If $\psi \omega^{-j}=-1$, then

$$
\lambda_{j}(J)=\frac{1}{9}\left(3 n+(-2)^{n+1}+2\right) .
$$


(3) If $\psi \omega^{-j} \neq \frac{1}{2}$ and $\psi \omega^{-j} \neq-1$, then

$$
\lambda_{j}(J)=\frac{k J_{n+1}-1+2 k J_{n} \psi \omega^{-j}}{2\left(\psi \omega^{-j}\right)^{2}+\psi \omega^{-j}-1} .
$$

Proof. Based on Lemma 2.1 and 1.6, we have:

(1) Suppose that $\psi \omega^{-j}=\frac{1}{2}$. Then,

$$
\begin{aligned}
\lambda_{j}(J) & =\sum_{i=0}^{n-1} J_{i+1}\left(\psi \omega^{-j}\right)^{i}=\frac{1}{3} \sum_{i=0}^{n-1}\left(2^{i+1}-(-1)^{i+1}\right)\left(\frac{1}{2}\right)^{i} \\
& =\frac{1}{3}\left(\sum_{i=0}^{n-1} 2+\sum_{i=0}^{n-1}\left(-\frac{1}{2}\right)^{i}\right)=\frac{1}{3}\left(2 n+\frac{1-\left(-\frac{1}{2}\right)^{n}}{\frac{3}{2}}\right) \\
& =\frac{2}{9}\left(3 n-\left(-\frac{1}{2}\right)^{n}+1\right) .
\end{aligned}
$$

(2) Suppose that $\psi \omega^{-j}=-1$. Then,

$$
\begin{aligned}
\lambda_{j}(J) & =\sum_{i=0}^{n-1} J_{i+1}\left(\psi \omega^{-j}\right)^{i}=\frac{1}{3} \sum_{i=0}^{n-1}\left(2^{i+1}-(-1)^{i+1}\right)(-1)^{i} \\
& =\frac{1}{3}\left(2 \sum_{i=0}^{n-1}(-2)^{i}+\sum_{i=0}^{n-1} 1\right)=\frac{1}{3}\left(2 \frac{1-(-2)^{n}}{3}+n\right) \\
& =\frac{1}{9}\left(3 n+(-2)^{n+1}+2\right) .
\end{aligned}
$$

(3) Suppose that $\psi \omega^{-j} \neq \frac{1}{2}$ and $\psi \omega^{-j} \neq-1$. Then, $\lambda_{j}(J)$ follows from 1.3 .

The following example illustrates the result of Theorem 2.2 .

Example 2.3. Let

$$
J=\operatorname{circ}\left\{\frac{1}{64}(1,1,3,5,11,21)\right\},
$$

i.e.,

$$
J=\left[\begin{array}{rrrrrr}
1 & 1 & 3 & 5 & 11 & 21 \\
\frac{21}{64} & 1 & 1 & 3 & 5 & 11 \\
\frac{11}{64} & \frac{21}{64} & 1 & 1 & 3 & 5 \\
\frac{5}{64} & \frac{11}{64} & \frac{21}{64} & 1 & 1 & 3 \\
\frac{3}{64} & \frac{5}{64} & \frac{11}{64} & \frac{21}{64} & 1 & 1 \\
\frac{1}{64} & \frac{3}{64} & \frac{5}{64} & \frac{11}{64} & \frac{21}{64} & 1
\end{array}\right]
$$

Since $n=6$ and $k=\frac{1}{64}$, i.e., $\psi=\frac{1}{2}$ and $\omega=\frac{1}{2}+i \frac{\sqrt{3}}{2}$, based on Theorem 2.2 it follows that

1) $\psi \omega^{0}=\frac{1}{2}$, so $\lambda_{0}(J)$ is obtained based on (1) of Theorem $2.2 \quad \lambda_{0}(J)=\frac{135}{32}$; 
2) $\psi \omega^{-j} \neq \frac{1}{2}$ and $\psi \omega^{-j} \neq 1$, for $j=\overline{1,5}$, so $\lambda_{j}(J)$, for $j=\overline{1,5}$, are obtained based on (3) of Theorem $2.2 \quad \lambda_{1,5}(J)=\frac{3}{64}(5 \pm i \sqrt{3}), \lambda_{2,4}(J)=\frac{7}{64}(3 \pm i \sqrt{3})$, $\lambda_{3}(J)=\frac{21}{32}$.

Since $|J|=\prod_{j=0}^{n-1} \lambda_{j}(J)$, it follows that $|J|=\frac{3^{7} 57^{4}}{2^{30}}$.

Remark 2.4 (In relation to Example 2.3). The determinant of

$$
J=\operatorname{circ}\left\{\frac{1}{64}(1,1,3,5,11,21)\right\}
$$

cannot be obtained using 1.4 .

Next, we determine the Euclidean norm of the matrix 1.2. The following formula will be used.

For all $x$,

$$
\sum_{i=1}^{n-1} i x^{i}=\frac{x-n x^{n}+(n-1) x^{n+1}}{(1-x)^{2}} .
$$

Theorem 2.5. Let $J$ be the matrix as in 1.2. The Euclidean norm of $J$ is

$$
\|J\|_{E}=\frac{1}{3} \sqrt{n\left[2^{n+2} J_{n}+n\right]+\left(|k|^{2}-1\right)\left[\frac{4}{9}\left[2-n 2^{n} j_{n}+(n-1) 2^{n+1} j_{n+1}\right]+\frac{(n-1) n}{2}\right]} .
$$

Proof. From the definition of the Euclidean norm of a matrix, using (1.6), 1.7), (1.8), and 2.1), we obtain:

$$
\begin{aligned}
\left(\|J\|_{E}\right)^{2}= & \sum_{i, j=1}^{n}\left|j_{i, j}\right|^{2} \\
= & n J_{1}^{2}+\left[(n-1)+|k|^{2}\right] J_{2}^{2}+\left[(n-2)+2|k|^{2}\right] J_{3}^{2}+\cdots+\left[1+(n-1)|k|^{2}\right] J_{n}^{2} \\
= & \sum_{i=0}^{n-1}(n-i) J_{i+1}^{2}+|k|^{2} \sum_{i=1}^{n-1} i J_{i+1}^{2} \\
= & n \sum_{i=0}^{n-1} J_{i+1}^{2}+\left(|k|^{2}-1\right) \sum_{i=1}^{n-1} i J_{i+1}^{2} \\
= & \frac{1}{9}\left[n\left[2^{n+2} J_{n}+n\right]+\left(|k|^{2}-1\right) \sum_{i=1}^{n-1} i\left[4^{i+1}-2(-2)^{i+1}+1\right]\right] \\
= & \frac{n}{9}\left[2^{n+2} J_{n}+n\right]+\frac{|k|^{2}-1}{9}\left[4 \frac{4-n 4^{n}+(n-1) 4^{n+1}}{9}\right. \\
& \left.+4 \frac{-2-n(-2)^{n}+(n-1)(-2)^{n+1}}{9}+\frac{(n-1) n}{2}\right] \\
= & \frac{n}{9}\left[2^{n+2} J_{n}+n\right] \\
& +\frac{|k|^{2}-1}{9}\left[\frac{4}{9}\left[2-n 2^{n} j_{n}+(n-1) 2^{n+1} j_{n+1}\right]+\frac{(n-1) n}{2}\right] .
\end{aligned}
$$


Therefore,

$$
\|J\|_{E}=\frac{1}{3} \sqrt{n\left[2^{n+2} J_{n}+n\right]+\left(|k|^{2}-1\right)\left[\frac{4}{9}\left[2-n 2^{n} j_{n}+(n-1) 2^{n+1} j_{n+1}\right]+\frac{(n-1) n}{2}\right]} .
$$

At the end of this paper, we determine the upper and lower bounds for the spectral norm of $J_{k}^{\circ-1}=\operatorname{circ}\left\{k_{k}\left(J_{1}^{-1}, J_{2}^{-1}, J_{3}^{-1}, \ldots, J_{n}^{-1}\right)\right\}$. The following lemma will be used.

Lemma 2.6 ([12]). Let $G=\left[g_{i, j}\right]$ and $H=\left[h_{i, j}\right]$ be matrices of order $m \times n$. Then,

$$
\|G \circ H\|_{2} \leq r_{1}(G) \cdot c_{1}(H),
$$

where $G \circ H=\left[g_{i, j} h_{i, j}\right]$ is the Hadamard product (or the Schur product) of the matrices $G$ and $H$,

$$
r_{1}(G)=\max _{1 \leq i \leq m} \sqrt{\sum_{j=1}^{n}\left|g_{i, j}\right|^{2}}, \quad \text { and } \quad c_{1}(H)=\max _{1 \leq j \leq n} \sqrt{\sum_{i=1}^{m}\left|h_{i, j}\right|^{2}} .
$$

We recommend the papers [11] and [18] for more information about the Hadamard product of matrices.

Theorem 2.7. Let $J_{k}^{\circ-1}=\operatorname{circ}\left\{{ }_{k}\left(J_{1}^{-1}, J_{2}^{-1}, J_{3}^{-1}, \ldots, J_{n}^{-1}\right)\right\}$.

(1) If $|k| \geq 1$, then

$$
3 \sqrt{\frac{n}{2 \cdot 4^{n}-(-2)^{n}-1}} \leq\left\|J_{k}^{\circ-1}\right\|_{2} \leq \sqrt{n\left(1+(n-1)|k|^{2}\right)} .
$$

(2) If $|k|<1$, then

$$
3|k| \sqrt{\frac{n}{2 \cdot 4^{n}-(-2)^{n}-1}} \leq\left\|J_{k}^{\circ-1}\right\|_{2} \leq n .
$$

Proof. From the definition of the Euclidean norm of a matrix, we have:

$$
\left\|J_{k}^{\circ-1}\right\|_{E}^{2}=\sum_{i=0}^{n-1}(n-i) \frac{1}{J_{i+1}^{2}}+|k|^{2} \sum_{i=1}^{n-1} i \frac{1}{J_{i+1}^{2}} .
$$

(1) If $|k| \geq 1$, then

$$
\begin{aligned}
\left\|J_{k}^{\circ-1}\right\|_{E}^{2} & \geq \sum_{i=0}^{n-1}(n-i) \frac{1}{J_{i+1}^{2}}+\sum_{i=1}^{n-1} i \frac{1}{J_{i+1}^{2}}=n \sum_{i=0}^{n-1} \frac{1}{J_{i+1}^{2}}=n \sum_{i=1}^{n} \frac{1}{J_{i}^{2}} \\
& \geq n \sum_{i=1}^{n} \frac{1}{J_{n}^{2}}=\left(\frac{n}{J_{n}}\right)^{2} \geq \frac{n^{2}}{J_{n} J_{n+1}}=\frac{9 n^{2}}{j_{2 n+1}-(-2)^{n}} \\
& =\frac{9 n^{2}}{2^{2 n+1}+(-1)^{2 n+1}-(-2)^{n}}=\frac{9 n^{2}}{2 \cdot 4^{n}-(-2)^{n}-1} .
\end{aligned}
$$


Therefore,

$$
\frac{\left\|J_{k}^{\circ-1}\right\|_{E}}{\sqrt{n}} \geq 3 \sqrt{\frac{n}{2 \cdot 4^{n}-(-2)^{n}-1}} .
$$

We conclude from 1.1 that

$$
\left\|J_{k}^{\circ-1}\right\|_{2} \geq 3 \sqrt{\frac{n}{2 \cdot 4^{n}-(-2)^{n}-1}} .
$$

Now, we shall obtain the upper bound for the spectral norm of $J_{k}^{\circ-1}$. Let $R$ and $S$ be the following matrices:

$$
R=\left[\begin{array}{ccccc}
\frac{1}{J_{1}} & \frac{1}{J_{2}} & \frac{1}{J_{3}} & \cdots & \frac{1}{J_{n}} \\
k & \frac{1}{J_{1}} & \frac{1}{J_{2}} & \cdots & \frac{1}{J_{n-1}} \\
k & k & \frac{1}{J_{1}} & \cdots & \frac{1}{J_{n-2}} \\
\vdots & \vdots & \vdots & \ddots & \vdots \\
k & k & k & \cdots & \frac{1}{J_{1}}
\end{array}\right] \text { and } S=\left[\begin{array}{ccccc}
1 & 1 & 1 & \cdots & 1 \\
\frac{1}{J_{n}} & 1 & 1 & \cdots & 1 \\
\frac{1}{J_{n-1}} & \frac{1}{J_{n}} & 1 & \cdots & 1 \\
\vdots & \vdots & \vdots & \ddots & \vdots \\
\frac{1}{J_{2}} & \frac{1}{J_{3}} & \frac{1}{J_{4}} & \cdots & 1
\end{array}\right]
$$

Then,

$$
r_{1}(R)=\max _{1 \leq i \leq n} \sqrt{\sum_{j=1}^{n}\left|r_{i, j}\right|^{2}}=\sqrt{\sum_{j=1}^{n}\left|r_{n, j}\right|^{2}}=\sqrt{1+(n-1)|k|^{2}}
$$

and

$$
c_{1}(S)=\max _{1 \leq j \leq n} \sqrt{\sum_{i=1}^{n}\left|s_{i, j}\right|^{2}}=\sqrt{\sum_{j=1}^{n}\left|s_{i, n}\right|^{2}}=\sqrt{n} .
$$

Since $J_{k}^{\circ-1}=R \circ S$, based on Lemma 2.6, we can write

$$
\left\|J_{k}^{\circ-1}\right\|_{2} \leq r_{1}(R) \cdot c_{1}(S)=\sqrt{n\left(1+(n-1)|k|^{2}\right)} .
$$

(2) If $|k|<1$, then

$$
\begin{aligned}
\left\|J_{k}^{\circ-1}\right\|_{E}^{2} & \geq \sum_{i=0}^{n-1}(n-i)|k|^{2} \frac{1}{J_{i+1}^{2}}+\sum_{i=1}^{n-1} i|k|^{2} \frac{1}{J_{i+1}^{2}}=n|k|^{2} \sum_{i=0}^{n-1} \frac{1}{J_{i+1}^{2}}=n|k|^{2} \sum_{i=1}^{n} \frac{1}{J_{i}^{2}} \\
& \geq n|k|^{2} \sum_{i=1}^{n} \frac{1}{J_{n}^{2}}=|k|^{2}\left(\frac{n}{J_{n}}\right)^{2} \geq|k|^{2} \frac{n^{2}}{J_{n} J_{n+1}}=|k|^{2} \frac{9 n^{2}}{j_{2 n+1}-(-2)^{n}} \\
& =|k|^{2} \frac{9 n^{2}}{2^{2 n+1}+(-1)^{2 n+1}-(-2)^{n}}=|k|^{2} \frac{9 n^{2}}{2 \cdot 4^{n}-(-2)^{n}-1} .
\end{aligned}
$$

Therefore,

$$
\frac{\left\|J_{k}^{\circ-1}\right\|_{E}}{\sqrt{n}} \geq 3|k| \sqrt{\frac{n}{2 \cdot 4^{n}-(-2)^{n}-1}}
$$


We conclude from 1.1 that

$$
\left\|J_{k}^{\circ-1}\right\|_{2} \geq 3|k| \sqrt{\frac{n}{2 \cdot 4^{n}-(-2)^{n}-1}} .
$$

Now, we shall obtain the upper bound for the spectral norm of $J_{k}^{\circ-1}$. Let $U$ and $V$ be the following matrices:

$$
U=\left[\begin{array}{ccccc}
1 & 1 & 1 & \cdots & 1 \\
\frac{k}{J_{n}} & 1 & 1 & \cdots & 1 \\
\frac{k}{J_{n-1}} & \frac{k}{J_{n}} & 1 & \cdots & 1 \\
\vdots & \vdots & \vdots & \ddots & \vdots \\
\frac{k}{J_{2}} & \frac{k}{J_{3}} & \frac{k}{J_{4}} & \cdots & 1
\end{array}\right] \text { and } V=\left[\begin{array}{ccccc}
\frac{1}{J_{1}} & \frac{1}{J_{2}} & \frac{1}{J_{3}} & \cdots & \frac{1}{J_{n}} \\
1 & \frac{1}{J_{1}} & \frac{1}{J_{2}} & \cdots & \frac{1}{J_{n-1}} \\
1 & 1 & \frac{1}{J_{1}} & \cdots & \frac{1}{J_{n-2}} \\
\vdots & \vdots & \vdots & \ddots & \vdots \\
1 & 1 & 1 & \cdots & \frac{1}{J_{1}}
\end{array}\right] .
$$

Then,

and

$$
r_{1}(U)=\max _{1 \leq i \leq n} \sqrt{\sum_{j=1}^{n}\left|u_{i, j}\right|^{2}}=\sqrt{\sum_{j=1}^{n}\left|u_{1, j}\right|^{2}}=\sqrt{n}
$$

$$
c_{1}(V)=\max _{1 \leq j \leq n} \sqrt{\sum_{i=1}^{n}\left|v_{i, j}\right|^{2}}=\sqrt{\sum_{j=1}^{n}\left|v_{i, 1}\right|^{2}}=\sqrt{n} .
$$

Since $J_{k}^{\circ-1}=U \circ V$, based on Lemma 2.6 we can write

$$
\left\|J_{k}^{\circ-1}\right\|_{2} \leq r_{1}(U) \cdot c_{1}(V)=n .
$$

The following example illustrates the result of Theorem 2.7 .

Example 2.8. Let $J_{k}^{\circ-1}=\operatorname{circ}\left\{{ }_{k}\left(J_{1}^{-1}, J_{2}^{-1}, J_{3}^{-1}, \ldots, J_{n}^{-1}\right)\right\}$.

The lower bounds for the spectral norm of $J_{k}^{\circ-1}$

a) for $|k| \geq 1$

\begin{tabular}{|l|c|}
\hline$n$ & $\left\|J_{k}^{\circ-1}\right\|_{2} \geq$ \\
\hline 2 & $\sqrt{\frac{2}{3}} \approx 0.81650$ \\
\hline 3 & $\sqrt{\frac{1}{5}} \approx 0.44721$ \\
\hline 4 & $\frac{2}{\sqrt{55}} \approx 0.26968$ \\
\hline 5 & $\sqrt{\frac{5}{231}} \approx 0.14712$ \\
\hline 6 & $\sqrt{\frac{2}{301}} \approx 0.08151$ \\
\hline 7 & $\sqrt{\frac{7}{3655}} \approx 0.04376$ \\
\hline
\end{tabular}

b) for $k=-\frac{1}{3}, \frac{1}{3}$

\begin{tabular}{|l|c|}
\hline$n$ & $\left\|J_{k}^{\circ-1}\right\|_{2} \geq$ \\
\hline 2 & $\frac{1}{3} \sqrt{\frac{2}{3}} \approx 0.27217$ \\
\hline 3 & $\frac{1}{3} \sqrt{\frac{1}{5}} \approx 0.14907$ \\
\hline 4 & $\frac{2}{3} \sqrt{\frac{1}{55}} \approx 0.08989$ \\
\hline 5 & $\frac{1}{3} \sqrt{\frac{5}{231}} \approx 0.04904$ \\
\hline 6 & $\frac{1}{3} \sqrt{\frac{2}{301}} \approx 0.02717$ \\
\hline 7 & $\frac{1}{3} \sqrt{\frac{7}{3655}} \approx 0.01459$ \\
\hline
\end{tabular}

c) for $k=-\frac{1}{2}, \frac{1}{2}$

\begin{tabular}{|l|c|}
\hline$n$ & $\left\|J_{k}^{\circ-1}\right\|_{2} \geq$ \\
\hline 2 & $\frac{1}{2} \sqrt{\frac{2}{3}} \approx 0.40825$ \\
\hline 3 & $\frac{1}{2} \sqrt{\frac{1}{5}} \approx 0.22361$ \\
\hline 4 & $\sqrt{\frac{1}{55}} \approx 0.13484$ \\
\hline 5 & $\frac{1}{2} \sqrt{\frac{5}{231}} \approx 0.07356$ \\
\hline 6 & $\frac{1}{2} \sqrt{\frac{2}{301}} \approx 0.04076$ \\
\hline 7 & $\frac{1}{2} \sqrt{\frac{7}{3655}} \approx 0.02188$ \\
\hline
\end{tabular}


The upper bounds for the spectral norm of $J_{k}^{\circ-1}$

a) for $k=-3,3$

\begin{tabular}{|c|c|}
\hline$n$ & $\left\|J_{k}^{\circ-1}\right\|_{2} \leq$ \\
\hline 2 & $\sqrt{20} \approx 4.47214$ \\
\hline 3 & $\sqrt{57} \approx 7.54983$ \\
\hline 4 & $\sqrt{112} \approx 10.58301$ \\
\hline 5 & $\sqrt{185} \approx 13.60147$ \\
\hline 6 & $\sqrt{276} \approx 16.61325$ \\
\hline 7 & $\sqrt{385} \approx 19.62142$ \\
\hline
\end{tabular}

b) for $k=-2,2$

\begin{tabular}{|c|c|}
\hline$n$ & $\left\|J_{k}^{\circ-1}\right\|_{2} \leq$ \\
\hline 2 & $\sqrt{10} \approx 3.16228$ \\
\hline 3 & $\sqrt{27} \approx 5.19615$ \\
\hline 4 & $\sqrt{52} \approx 7.21110$ \\
\hline 5 & $\sqrt{85} \approx 9.21954$ \\
\hline 6 & $\sqrt{126} \approx 11.22497$ \\
\hline 7 & $\sqrt{175} \approx 13.22876$ \\
\hline
\end{tabular}

c) for $|k|<1$

\begin{tabular}{|c|c|}
\hline$n$ & $\left\|J_{k}^{\circ-1}\right\|_{2} \leq$ \\
\hline 2 & 2 \\
\hline 3 & 3 \\
\hline 4 & 4 \\
\hline 5 & 5 \\
\hline 6 & 6 \\
\hline 7 & 7 \\
\hline
\end{tabular}

\section{Conclusion}

In this paper, we considered the matrix

$$
J=\operatorname{circ}\left\{k\left(J_{1}, J_{2}, \ldots, J_{n}\right)\right\},
$$

where $J_{n}$ is the $n^{\text {th }}$ Jacobsthal number and $k$ is a nonzero complex number, and obtained the formulae for the eigenvalues of such matrix improving the result of [26, Theorem 7]. The obtained formulae (for the eigenvalues of such matrix) showed that the result of [14, Theorem 10] (i.e., [26, Theorem 8]) is not always applicable. We also obtained the Euclidean norm of such matrix and bounds for the spectral norm of a $k$-circulant matrix whose first row is $\left(J_{1}^{-1}, J_{2}^{-1}, \ldots, J_{n}^{-1}\right)$.

For $k=1$, we obtain the results (the eigenvalues and the Euclidean norm) related with a circulant matrix whose first row is $\left(J_{1}, J_{2}, \ldots, J_{n}\right)$. Hence, this paper complements the paper 2] where the authors considered a circulant matrix whose first row is $\left(J_{1}, J_{2}, \ldots, J_{n}\right)$ and obtained the determinant (without using the formulae for the eigenvalues) and the inverse of such matrix.

From the well known fact that the eigenvalues of an upper triangular matrix are the diagonal entries, it follows that the eigenvalues of $J$ for $k=0$ (a semicirculant matrix) are $\lambda_{j}(J)=1, j=\overline{0, n-1}$. The Euclidean norm of such matrix can be obtained from 2.2, i.e., $k$ can be equal to 0 in 2.2. The upper and lower bounds for the spectral norm of a semicirculant matrix whose first row is $\left(J_{1}^{-1}, J_{2}^{-1}, \ldots, J_{n}^{-1}\right)$ can be obtained from (2.4).

\section{ACKNOWLEDGEMENT}

We would like to thank the anonymous reviewer for his careful reading of our manuscript, his comments and his useful suggestions that improved the quality of the manuscript. 


\section{REFERENCES}

[1] D. Bertaccini and M. K. Ng, Skew-circulant preconditioners for systems of LMF-based ODE codes. In: Numerical analysis and its applications (Rousse, 2000), 93-101, Lecture Notes in Comput. Sci., 1988, Springer, Berlin, 2001. MR 1938408

[2] D. Bozkurt and T-Y. Tam, Determinants and inverses of circulant matrices with Jacobsthal and Jacobsthal-Lucas numbers, Appl. Math. Comput. 219 (2012), no. 2, 544-551. MR 2956985

[3] D. Bozkurt and T-Y. Tam, Determinants and inverses of $r$-circulant matrices associated with a number sequence, Linear Multilinear Algebra 63 (2015), no. 10, 2079-2088. MR 3378017.

[4] R. E. Cline, R. J. Plemmons and G. Worm, Generalized inverses of certain Toeplitz matrices, Linear Algebra Appl. 8 (1974), no. 1, 25-33. MR 0347844

[5] R. L. Ellis and D. C. Lay, Factorization of finite rank Hankel and Toeplitz matrices, Linear Algebra Appl. 173 (1992), 19-38. MR 1170502

[6] M. C. Gouveia, Generalized invertibility of Hankel and Toeplitz matrices, Linear Algebra Appl. 193 (1993), 95-106. MR 1240274.

[7] R. M. Gray, Toeplitz and circulant matrices: A review, Found. Trends Commun. Inf. Theory 2 (2006), no. 3, 155-239.

[8] E. J. Hannan, Time Series Analysis, Methuen, London, 1960. MR 0114281

[9] C. He, J. Ma, K. Zhang and Z. Wang, The upper bound estimation on the spectral norm of $r$-circulant matrices with the Fibonacci and Lucas numbers, J. Inequal. Appl. 2015, 2015:72, 10 pp. MR 3316758

[10] A. F. Horadam, Jacobsthal representation numbers, Fibonacci Quart. 34 (1996), no. 1, 4054. MR 1371475

[11] R. A. Horn, The Hadamard product. In: Matrix theory and applications (Phoenix, AZ, 1989), 87-169, Proc. Sympos. Appl. Math., 40, AMS Short Course Lecture Notes, Amer. Math. Soc., Providence, RI, 1990. MR 1059485.

[12] R. A. Horn and C. R. Johnson, Topics in Matrix Analysis, Cambridge Univ. Press, Cambridge, 1991. MR 1091716

[13] I. S. Iohvidov, Hankel and Toeplitz Matrices and Forms: Algebraic Theory, Birkhäuser, Boston, 1982. MR 0677503

[14] Z. Jiang, J. Li and N. Shen, On the explicit determinants and singularities of $r$-circulant and left $r$-circulant matrices with some famous numbers, WSEAS Trans. Math. 12 (2013), no. 3, 341-351.

[15] E. Gokcen Kocer, Circulant, negacyclic and semicirculant matrices with the modified Pell, Jacobsthal and Jacobsthal-Lucas numbers, Hacet. J. Math. Stat. 36 (2007), no. 2, 133-142. MR 2411631.

[16] F. Koken and D. Bozkurt, On the Jacobsthal numbers by matrix methods, Int. J. Contemp. Math. Sci. 3 (2008), no. 13-16, 605-614. MR 2484951

[17] G. Labahn and T. Shalom, Inversion of Toeplitz matrices with only two standard equations, Linear Algebra Appl. 175 (1992), 143-158. MR 1179345

[18] S. Liu and G. Trenkler, Hadamard, Khatri-Rao, Kronecker and other matrix products, Int. J. Inf. Syst. Sci. 4 (2008), no. 1, 160-177. MR 2401772

[19] J. N. Lyness and T. Sørevik, Four-dimensional lattice rules generated by skew-circulant matrices, Math. Comput. 73 (2004), no. 245, 279-295. MR 2034122

[20] M. K. Ng, Circulant and skew-circulant splitting methods for Toeplitz systems, J. Comput. Appl. Math. 159 (2003), no. 1, 101-108. MR 2022320.

[21] B. Radičić, On k-circulant matrices (with geometric sequence), Quaest. Math. 39 (2016), no. 1, 135-144. MR 3483362

[22] B. Radičić, On $k$-circulant matrices with arithmetic sequence, Filomat 31 (2017), no. 8, 2517-2525. MR 3637047.

[23] S. Q. Shen and J. M. Cen, On the bounds for the norms of $r$-circulant matrices with the Fibonacci and Lucas numbers, Appl. Math. Comput. 216 (2010), no. 10, 2891-2897. MR 2653105 
[24] W. Sintunavarat, The upper bound estimation for the spectral norm of $r$-circulant and symmetric $r$-circulant matrices with the Padovan sequence, J. Nonlinear Sci. Appl. 9 (2016), no. 1, 92-101. MR 3392176

[25] W. F. Trench, An algorithm for the inversion of finite Toeplitz matrices, J. Soc. Indust. Appl. Math. 12 (1964), no. 3, 515-522. MR 0173681.

[26] Y. Yazlik and N. Taskara, On the norms of an $r$-circulant matrix with the generalized $k$ Horadam numbers, J. Inequal. Appl. 2013, 2013:394, 8 pp. MR 3110742

[27] G. Zhao, The improved nonsingularity on the $r$-circulant matrices in signal processing. In: Proceedings, 2009 International Conference on Computer Technology and Development (Kota Kinabalu, Malaysia), 564-567, IEEE, 2009.

[28] G. Zielke, Some remarks on matrix norms, condition numbers, and error estimates for linear equations, Linear Algebra Appl. 110 (1988), 29-41. MR 0964206.

\section{B. Radičić}

Singidunum University, Belgrade, Serbia

bradicic@singidunum.ac.rs

Received: March 6, 2018

Accepted: January 22, 2019 